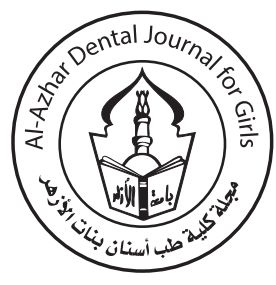

\title{
Marginal Fit Evaluation of Zirconia and Polyetheretherketone Inlay Retained Fixed Partial Dentures Fabricated with CAD-CAM
}

\author{
Jannat W. Hassan ${ }^{1 *}$, Atef F. Ahmed ${ }^{2}$, Walaa O. El-Mekakwi .
}

Codex : 03/22.01

azhardentj@azhar.edu.eg

http://adjg.journals.ekb.eg

DOI: $10.21608 /$ adjg.2021.48254.1318

Restorative Dentistry

(Removable Prosthodontics, Fixed

Prosthodontics, Endodontics, Dental Biomaterials, Operative Dentistry)

\section{KEYWORDS}

Zirconia, PEEK, Marginal Fit, IRFPD, CAD-CAM.

\begin{abstract}
Purpose: The purpose of this study was to compare marginal fit of Zirconia and Polyetheretherketone (PEEK) inlay retained fixed partial dentures (IRFPD) fabricated by using a computer-aided design and computer-aided manufacturing (CAD-CAM) system. Materials and Methods: Sound Mandibular second premolar and mandibular second molar were selected to simulate a clinical situation of a missing mandibular first molar and embedded in an epoxy resin (master model). Both teeth received a standard preparation of IRFPD with respect to all-ceramic preparation design parameters. The master model was duplicated into 18 epoxy resin models for standardization during testing procedure, and 18 IRFPDs were fabricated with CAD-CAM system and divided into 2 groups according to materials type $(n=9)$; Group 1: 3-mol yttria-stabilized tetragonal zirconia polycrystal (3Y-TZP), Group 2: PEEK. Marginal Discrepancy of all bridges was tested before and after cementation with RelyX U200 self-adhesive resin cement (SARC) by using an optical digital microscope with the aid of an image analysis system. Results: Significantly lower marginal gap values were obtained in Zirconia group before and after cementation in comparison to PEEK group. There was also a significant increase of marginal gap after cementation in both groups, with higher mean in Zirconia group. Conclusion: The margins of IRFPDs performed well with recommended bonding protocols for both materials and present a viable treatment option for replacing a missing single posterior tooth. Both materials presented clinically accepted results of marginal discrepancy less than $120 \mu \mathrm{m}$. Further studies simulating clinical conditions are needed to understand how they perform in function.
\end{abstract}

* Paper extracted from Master Thesis titled "Evaluation of Marginal fit and Retention of Zirconia and PEEK Inlay retained fixed partial dentures fabricated with CAD-CAM"

1. Teaching assistant of Fixed Prosthodontics department, Faculty of Oral and Dental Medicine, Badr University in Cairo, Cairo, Egypt.

2. Professor of Crown and Bridge, Faculty of Oral and Dental Medicine for Girls, Al-Azhar University, Cairo, Egypt.

3. Assistant Professor of Crown and Bridge, Faculty of Oral and Dental Medicine for Girls, Al-Azhar University, Cairo, Egypt.

*Corresponding author email: jenyel7ariry@gmail.com 


\section{INTRODUCTION}

Various treatment modalities are available to replace missing posterior teeth; metal- ceramic, all ceramic, direct or indirect fiber reinforced composite fixed partial dentures (FPDs), the conventional removable prosthesis and the implant supported restorations. Implant supported restorations are a more conservative approach to single-tooth replacement. However, this line of treatment has its own limitations, which sometimes makes FPDs the only available choice ${ }^{(1)}$.

FPDs require considerable reduction of tooth structure; therefore, some patients and clinicians are reluctant to use it especially in healthy, young dentition with large pulp chambers ${ }^{(2)}$. The IRFPD enables the original tooth anatomy to be reproduced while allowing for functionality, aesthetics, and preservation of the tooth structure making IRFPD a valid therapeutic option to replace a single missing tooth.

The increasing demand for esthetic restorations that can also serve as durable long-term restorations capable of withstanding the patients' masticatory forces and adapting to the oral environment coincided with the introduction of ceramic optimized polymers (Ceromers), fiber-reinforced composites (FRC) technology and all-ceramic systems; including ceramics with a high content of glass particles (i.e. lithium disilicate, glass-infiltrated zirconia or alumina), and high strength ceramics (densely sintered zirconia/alumina polycrystals) and Zirconia. Leading to the potential for fabrication of metal free restorations with high durability and good esthetics ${ }^{(3)}$.

A clinical option was introduced using monolithic zirconia in fabrication of IRFPDs. It was concluded that CAD-CAM monolithic zirconia IRFDP allows a minimally invasive approach for single-tooth substitution, and thus can be used as an alternative to a full-coverage FDP or an implantsupported crown ${ }^{(4,5)}$.

A new material group of high-performance polymers which was recently introduced for fabrication of fixed partial dentures is BioHPP (High-Performance Polymer). BioHPP is based on ceramicsstrengthened polyetheretherketone (PEEK). It has been used successfully in human medicine for more than 30 years as an implant material (finger prostheses, intervertebral discs and hip joint prostheses. It offers excellent biocompatibility and has a bone-like flexibility which makes it ideal for use in implant-supported dental prosthetics (OffPeak Effect) as the chewing forces are cushioned ${ }^{(6)}$. Thanks to strengthening with special ceramic filler, optimized mechanical properties of BioHPP have been created for dental use in the crown and bridge $\operatorname{area}^{(7)}$.

The accuracy of restoration's margins is an important parameter in the success of extra and intra coronal restorations. Marginal and internal gap size can influence longevity, wear, discoloration, leakage, degradation of the luting agent, and the ability of the restoration to withstand loading ${ }^{(8,9)}$.

\section{MATERIALS AND METHODS}

\section{Sample size calculation}

A power analysis was designed to have adequate power to apply a two-sided statistical test of the research hypothesis (null hypothesis) that there is no difference between both tested groups. According to the results of Pilo, R., et al. an effect size (d) of (1.47) was calculated and by adopting an alpha $(\alpha)$ level of $0.05(5 \%)$, and a beta $(\beta)$ level of 0.20 $(20 \%)$ i.e. power $=80 \%$; the predicted sample size (n) was found to be a total of (18) samples i.e. (9) samples per group ${ }^{(10)}$. Sample size calculation was performed using $G^{*}$ Power version 3.1.9.4

To conduct the present study, 2 freshly extracted human teeth $\left(1\right.$ sound mandibular $2^{\text {nd }}$ premolar and 1 mandibular $2^{\text {nd }}$ molar), were selected. The teeth were thoroughly cleaned using an ultrasonic cleaning device, dried gently and stored in $0.1 \%$ thymol solution at room temperature. 
Ethical approval for the use of extracted human teeth was obtained in accordance with guidelines from research ethics committee of faculty of dental medicine (Girls' Branch), Al Azhar University (18-027).

\section{Model Construction:}

A master model, formed of a $2^{\text {nd }}$ mandibular premolar and a $2^{\text {nd }}$ mandibular molar embedded in an epoxy resin block with $11 \mathrm{~mm}$ inter-abutment distance representing missing mandibular $1^{\text {st }}$ molar, was constructed according to the following procedure:

Putty consistency of Zeta plus (3M ESPE, Seefeld, Germany) was mixed and placed in the premolar-molar region of a pre-fabricated plastic dental arch (Elbanna, Alexandria, Egypt). By the aid of a dental surveyor (Surveyor B2, Bio-Art, São Carlos, Brazil), the teeth were embedded into the impression mix in a parallel position and at the same occlusal level. After rubber base setting, the mandibular 1st molar was removed, the area filled with wax, and an edentulous contour was made. An occlusal index was taken by putty consistency rubber base. The mandibular 2nd premolar and 2 nd molar were removed from the plastic dental arch and placed into their corresponding positions in the index. The index was then boxed with remodeling wax and sealed. Afterwards, epoxy resin (Crystal Clear Epoxy Resin, East Coast Resin. USA) was mixed following manufacturer's instructions and poured into the boxed index on a vibrator to remove any air bubbles. After complete setting, the boxing was removed, and the rubber base index was separated from the epoxy resin model and the model was checked for any defects under a magnifier.

\section{Teeth Preparation:}

The teeth were prepared according to the standard preparation guidelines for class II inlay with general principles for ceramic inlay restoration, with the aid of a Centroid milling machine (CNC, Milling machine, USA). The Occlusal cavity measurements were $(3 \pm 1 \mathrm{~mm} / 4 \pm 1 \mathrm{~mm})$ buccolingually and $(5 \pm 1 \mathrm{~mm} / 7 \pm 1 \mathrm{~mm})$ mesiodistally for the premolar/ molar abutments, respectively. The depth was adjusted at $2 \mathrm{~mm}$ measured from the central groove. The proximal cavity was extended with flared buccal and lingual walls $(4 \mathrm{~mm} / 5 \mathrm{~mm})$ for the premolar/molar abutments, respectively. The proximal box measured $4 \mathrm{~mm}$ in length and $1.5 \mathrm{~mm}$ in depth. Occlusal divergence angle was set at $10^{\circ}-12^{\circ}$. Cavo-surface margins were finished in butt joints with no bevels. Internal line and point angles were rounded.

\section{Model Duplication:}

A half-arch impression was made for the master model with prepared teeth using polyvinylsiloxane impression material. After setting and separation from the master model, the impression was inspected for any tears or defects, which if observed the impression was retaken. Dental epoxy resin material (Die epoxy type 8000 system, American dental supply Inc.) was blended according to the manufacturer's instructions and was poured into the half-arch impression on a vibrator to remove any air bubbles and checked for any defects after setting. The procedure was repeated to obtain 18 epoxy resin models accurately similar to the master model to ensure standardization during testing procedure.

\section{Samples' Grouping:}

The constructed models $(\mathrm{n}=18)$ were randomly divided into 2 groups $(n=9)$ according to material type: Group (1): 3Y-TZP Katana STML (Kuraray Noritake Dental Inc, Tokyo, Japan), Group (2): Juvora PEEK (Straumann AG, Basel, Switzerland).

\section{IRFPDs fabrication:}

Nine Zirconia IRFPDs were fabricated according to the following procedure: Each constructed model was sprayed with light reflecting powder (Occutec, Scanspray, USA), and secured on the tray for taking the optical impression. The fully anatomical IRFPD design was designed according to the manufacturer's directions and 
software recommendations (Exocad GmbH, Darmstadt, Germany) including; a virtual cement space of $50 \mu \mathrm{m}$ was set $0.5 \mathrm{~mm}$ short of the margins, modified ridge-lap pontic and $4 \times 4 \mathrm{~mm}^{2}$ connector dimensions with rounded $0.6 \mathrm{~mm}$ radius of curvature at the gingival area. Data was sent to a 5-axis milling machine Roland DWX50 (Roland DG Corporation. Japan) to fabricate the bridges using a $14 \mathrm{~mm}$ blank. Afterwards, the bridges were placed on their occlusal surface inside the ceramic sintering tray, which was filled with sintering beads and then sintered in the HTC furnace (High-Temperature with program Control Unit), according to manufacturer instructions. Nine PEEK IRFPDs were constructed from a PEEK blank $(12 \mathrm{~mm})$ using the same procedure and system for scanning, designing and milling.

\section{Cementation procedure}

Before cementation, the restorations were tried in on the abutment teeth to check for proper seating. Surface treatment of epoxy resin models was performed by sandblasting the fitting surfaces with $50 \mu \mathrm{m} \mathrm{Al}_{2} \mathrm{O}_{3}$ at 2 bar pressure for 10 seconds at $10 \mathrm{~mm}$ distance. The internal surfaces of inlay retainers of Zirconia and PEEK IRFPDs were abraded with $\mathrm{Al}_{2} \mathrm{O}_{3}(50 \mu \mathrm{m}$ at 2 bar pressure /50 $\mu \mathrm{m}$ at 4 bar pressure respectively) at a $10 \mathrm{~mm}$ distance for 15 seconds using a special holder to standardize the distance.

The cementation was done using RelyX-U200 SARC (RXU200; 3M ESPE, Seefeld, Germany) according to manufacturer's recommendations. The resin cement was dispensed onto the fitting surface of the IRFPDs, which were slowly seated on the abutment teeth using adequate finger pressure allowing excess cement to seep out. The excess cement was removed by sharp scaler after 2 seconds of curing using a sharp dental explorer, then immediately placed under a loading device of $10 \mathrm{Kg}$. Light curing of 40 seconds from each side was done and the IRFPD was left in place for 5 minutes to ensure complete polymerization of the resin.

\section{Marginal Accuracy test}

The marginal gap width pre- and post-cementation was measured with a digital image-analysis system using digital photographs acquired with optical digital microscope at a fixed magnification of 50X (fig. 1). Marginal gap was expressed in pixels; thus, system calibration was performed to convert the pixels into absolute units of micron by comparing a ruler with a scale. The marginal gap was measured at 22 measuring locations in each IRFPD at an approximately constant interval along the margins of the restoration.

\section{Statistical analysis}

Numerical data was represented as mean and standard deviation (SD) values. Shapiro-Wilk's test was used to test for normality. Homogeneity of variances was tested using Levene's test. Independent and paired t-test were used to analyze inter and intragroup comparisons respectively. The significance level was set at $\mathrm{p} \leq 0.05$ within all tests. Statistical analysis was performed with $\mathrm{R}$ statistical analysis software version 4.0.3 for Windows.

\section{RESULTS}

There were no outliers, as assessed by boxplot of grouped data. The data was normally distributed, as assessed by Shapiro-Wilk's test of normality $(p>0.05)$ and there was homogeneity of variances ( $p>0.05)$ as assessed by Levene's test. Before and after cementation, significantly higher marginal gap values were measured in PEEK samples $(\mathrm{p}<0.001)$. In samples of both groups, there was a significant increase of marginal gap after cementation $(p<0.001)$. Percentage change of marginal gap after cementation was significantly higher in Zirconia samples $(\mathrm{p}<0.001)$. Results of student t-tests were summarized in (table 1). Mean and standard deviation values for marginal gap percentage change after cementation were presented in (fig. 2). 

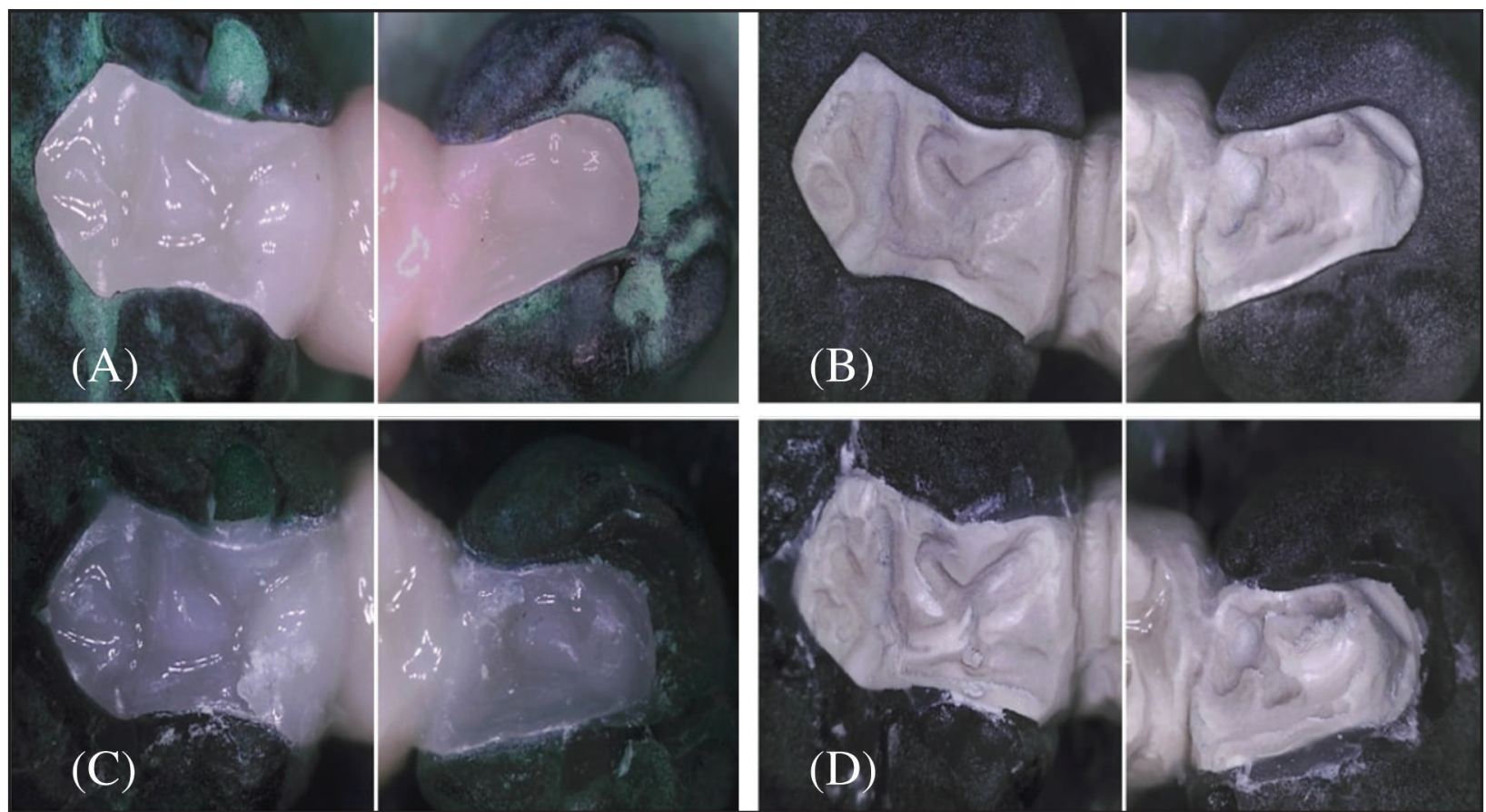

Figure (1) Marginal gap evaluation with a digital microscope $(\times 50)$ a. Zirconia IRFPD before cementation. b. PEEK IRFPD before cementation. c. Zirconia IRFPD after cementation. d. PEEK IRFPD after cementation.

Table (1): Summary of student t-test results

\begin{tabular}{|c|c|c|c|c|c|c|}
\hline \multirow{2}{*}{ Time } & \multicolumn{2}{|c|}{ Marginal gap $($ Mean $\pm S D)$} & \multirow{2}{*}{ Mean difference $[95 \% \mathrm{CI}]$} & \multirow{2}{*}{ Cohen's d } & \multirow{2}{*}{ t-value } & \multirow{2}{*}{$p$-value } \\
\hline & Zirconia & PEEK & & & & \\
\hline Before cementation & $30.05 \pm 1.89^{\mathrm{B}}$ & $63.58 \pm 2.28^{\text {в }}$ & $-33.53[-36.24:-30.82]$ & -16.00 & -27.72 & $<0.001 *$ \\
\hline After cementation & $46.18 \pm 1.99^{\mathrm{A}}$ & $78.05 \pm 3.29^{\mathrm{A}}$ & $-31.86[-35.47:-28.26]$ & -11.71 & -20.28 & $<0.001 *$ \\
\hline Change (\%) & $54.06 \pm 9.05$ & $22.76 \pm 2.62$ & $31.3[21.82: 40.79]$ & 4.70 & 8.13 & $<0.001 *$ \\
\hline
\end{tabular}

Means with different superscript letters in the same vertical column are statistically significant different;

*significant $(p<0.05)$

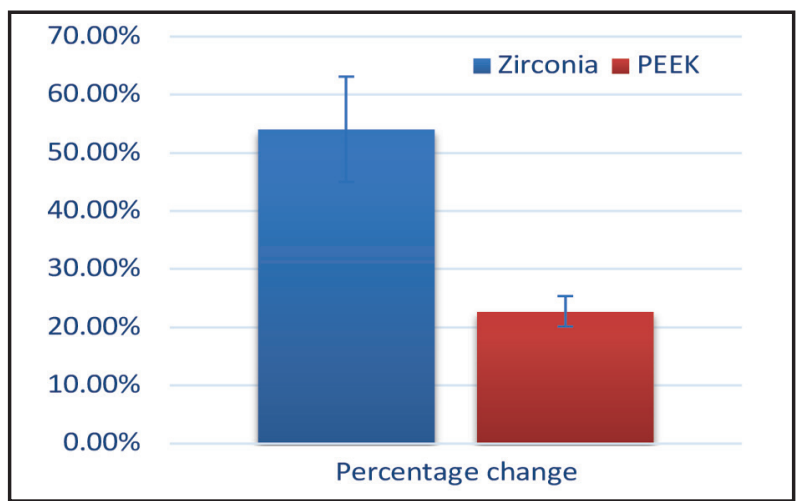

Figure (2) Bar chart showing mean and standard deviation values for marginal gap percentage change after cementation 


\section{DISCUSSION}

Conservative esthetic restorative dentistry has become an essential component in modern clinical practice. Accordingly, it would be desirable to restore a missing tooth with an inlay-retained FPD instead of a full coverage FPD; especially when sufficient sound tooth structure is available and the patient has favorable occlusion and good oral hygiene ${ }^{(11)}$.

Translucent zirconia was introduced to match patient's esthetic expectations, especially in the posterior zone, without compromising its flexural strength, therefore, in the current study it was decided to use monolithic translucent Zirconia for the construction of the IRFPDs to eliminate the possibility of failure due to chipping or delamination in the restorations ${ }^{(12)}$. In this study PEEK IRFPDs were also, designed to full contour in order to exclude the risk of veneering material chipping during testing procedures. The connector dimensions were $4 \times 4 \mathrm{~mm}^{2}$ to enhance fracture resistance of the restoration $^{(13)}$.

Marginal precision is considered a crucial factor in the success of restorations. Poor fitting margins may lead to cement dissolution, tooth sensitivity, recurrent caries, pulp exposure, and periodontal problems ${ }^{(14)}$.

With the introduction of different materials with different mechanical, physical and microstructural properties; it was important to experiment constructing posterior IRFPD using these innovative materials. Accordingly, the aim of this study was to determine that the marginal accuracy of both materials is within clinically acceptable range fabricated with CAD/CAM system.

Marginal gap was measured before and after cementation of the IRFPDs in the present study because the cement layer may increase the marginal gap uncontrollably and unevenly depending on the cement type, viscosity, and cementation technique. All samples were cemented using RelyX U200 selfadhesive resin cement with $50-1 \mathrm{~m}$ virtual cement space which causes only slight elevation of the crown within the limits of clinical acceptability ${ }^{(10)}$.

The marginal gap measurement was performed using an optical digital microscope; the most frequently method used to quantify the accuracy of restorations' fit and is considered the most convenient, accurate, easy, and rapid method for determining the marginal gap distance ${ }^{(11)}$.

The results of the current study showed significant difference between the 2 groups pre- and post-cementation. The mean marginal gap values were $30.05 \pm 1.89 \mu \mathrm{m}$ for the Zirconia group, 63.58 $\pm 2.28 \mu \mathrm{m}$ for the PEEK group pre-cementation, $46.18 \pm 1.99 \mu \mathrm{m}$ for the Zirconia group, 78.05 \pm 3.29 $\mu \mathrm{m}$ for the PEEK group post-cementation

These findings were consistent the mean AMG values of $35 \mu \mathrm{m}$ pre-cementation and $72 \mu \mathrm{m}$ postcementation of zirconia crown copings and with the mean marginal gap value of $43 \mu \mathrm{m}$ for $3 \mathrm{Y}$-TZP copings obtained in other studies which are all within the limits of clinical acceptability of $120 \mu \mathrm{m}$ set for cast gold copings but also widely accepted for all-ceramic crowns ${ }^{(10,15)}$. However, it was in contrast to better marginal and internal adaptation of PEEK compared to zirconia as reported in another study, which could be due to different types of blocks and CAD-CAM systems and the unpredictable sintering shrinkage of zirconia as proposed by the authors ${ }^{(16)}$.

There was a statistically significant difference in the mean marginal gaps $(54.06 \pm 9.05,22.76 \pm 2.62)$ in both groups between pre-cementation and postcementation. A study reporting the mean AMG before and after cementation $34.83 \pm 17.4 \mu \mathrm{m}$ and $72.00 \pm 31.22 \mu \mathrm{m}$, is in accordance with the results obtained in this study which was proposed to be a result of multiple factors including virtual cement space, cement type or variations in fabrication ${ }^{(10)}$. On the contrary, results obtained from another study with marginal discrepancy of all frameworks; PEEK and zirconia decreasing after cementation, was attributed to the compensation of small gaps by resin cement ${ }^{(16)}$. 


\section{CONCLUSION}

Within the limitations of this in-vitro study on the marginal fit of Zirconia and PEEK IRFPDs, the following conclusions can be drawn:

- The margins of IRFPDs performed well with recommended bonding protocols for both materials.

- All marginal gap values recorded were within the clinically acceptable range of $120 \mu \mathrm{m}$, with zirconia presenting significantly better values.

- Further in-vitro and vivo studies are needed to evaluate the marginal integrity of IRFPDs to understand how they perform in function.

\section{CONFLICT OF INTEREST}

None declared.

\section{FUNDING}

No funding was received for this study.

\section{REFERENCES}

1. Duqum I, Barker S, Marshall E, Wang R, Preisser J, Khan A. The effect of single tooth implant restorations on the survival, morbidity, pulpal, and periapical health of adjacent teeth: A chart review. Clinical Implant Dentistry and Related Research. 2018;20

2. Jung RE, Zembic A, Pjetursson BE, Zwahlen M, Thoma DS. Systematic review of the survival rate and the incidence of biological, technical and esthetic complications of single crowns on implants reported in longitudinal studies with a mean follow-up of 5 years. Clin. Oral Implants Res. 2012;23, 2-21

3. Thompson MC, Thompson KM, Swain M. The all-ceramic, inlay supported fixed partial denture. Part 1. Ceramic inlay preparation design: a literature review. Aust Dent J. 2010;55:120-7

4. Dapieve K, Guilardi L, Silvestri T, Rippe M, Pereira G, Valandro L. Mechanical performance of Y-TZP monolithic ceramic after grinding and aging: Survival estimates and fatigue strength. Journal of the Mechanical Behavior of Biomedical Materials, 2018;87:288-295.

5. Pereira G, Guilardi L, Dapieve K, Kleverlaan C, Rippe M, Valandro L. Translucent zirconia: Mechanical reliability, fatigue strength, survival and phase analysis. Dental Materials, 2018;34:e36.
6. Rzanny A, Göbel R, Fachet M. BIOHPP Summary of results for material tests. Quintessenz Zahntech MAG 2013;39:2-10.

7. Siewert B and Parra M: A new group of material in dentistry. Peek as a framework material used in 12-piece implantsupported bridges. Z Zahnarzt Implantol 2013;29:148-59.

8. Ahmed WM, Shariati B, Gazzaz AZ, Sayed ME, Carvalho RM. Fit of tooth-supported zirconia single crowns, A systematic review of the literature. Clin Exp Dent Res. 2020;1-17.

9. Ji M, Park J, Park S, Yun K, Oh G, Lim H. Evaluation of marginal fit of $2 \mathrm{CAD}-\mathrm{CAM}$ anatomic contour zirconia crown systems and lithium disilicate glass-ceramic crown. The Journal of Advanced Prosthodontics, 2015;7:271-7.

10. Pilo R, Folkman M, Arieli A, Levartovsky S. Marginal Fit and Retention Strength of Zirconia Crowns Cemented by Selfadhesive Resin Cements. Oper Dent. 2018;43:151-161.

11. El-khodary NA, Katamesh HA, Halim $\mathrm{CH}$. In vitro Fracture Resistance and Marginal Accuracy of Zirconia Inlay-retained Fixed Partial Denture: A Systematic Review. Indian J Sci Technol 2016;9:1-7.

12. Miura S, Kasahara S, Yamauchi S, Okuyama Y, Izumida A, Aida J et al Clinical evaluation of zirconia-based all-ceramic single crowns: an up to 12-year retrospective cohort study. Clin Oral Investig. 2018;22:697-706.

13. Hamza TA, Attia MA, El-Hossary MM, Mosleh IE, Shokry TE, Wee AG. Flexural strength of small connector designs of zirconia-based partial fixed dental prostheses. J Prosthet Dent 2016:1-6.

14. Aldegheishem A, Ioannidis G, Att W, Petridis H. Success and survival of various types of all-ceramic single crowns: A critical review and analysis of studies with a mean follow-up of 5 years or longer. Int J Prosthodont 2017;30:168-81.

15. Mazen A. Attia, Tamer E. Shokry, Effect of different fabrication techniques on the marginal precision of polyetheretherketone single-crown copings, J Prosthet Dent, 2020;124:565

16. Zeighami S, Ghodsi S, Sahebi M, Yazarloo S. Comparison of Marginal Adaptation of Different Implant-Supported Metal-Free Frameworks Before and After Cementation. Int J Prosthodont. 2019;32:361-363. 